\title{
Erector Spinae Plane Block- An Analgesic Technique for Laparoscopic Liver Resection: A Case Series
}

\section{Cristiano Piangatelli ${ }^{1}$, Diego Tavoletti ${ }^{2}$, Elisa Bottari ${ }^{2}$, Elisabetta Rosanò ${ }^{1}$, Ivana Lisanti ${ }^{1}$, Elisabetta Cerutti ${ }^{1}$}

\section{Abstract}

Introduction: Postoperative pain after liver surgery can be significant and yet difficult to manage. Epidural analgesia is widely used for pain relief after liver surgery. However there has been a progressive reduction in the use of epidural analgesia within an enhanced recovery program. The erector spinae plane block is a recently described regional anesthetic technique for providing abdominal analgesia when performed at the level of the $\mathrm{T} 7$ transverse process. Its mechanism of action is not yet clear, despite this providing somatic and visceral analgesia during surgery.

Cases presentation: We report five patients undergoing laparoscopic liver resection in which the somatic and visceral intraoperative pain were covered by the right erector spinae plane block. In addition, a transversus abdominis plane plus oblique subcostal transversus abdominis plane blocks were performed on the left hemisome to cover the somatic pain due to skin incision and laparoscopic ports insertion. For postoperative pain relief, a catheter was inserted during the execution of the erector spinae plane block and a levobupivacaine infusion was maintained for $36 \mathrm{hr}$ through it.

Conclusions: A continuous right erector spinae plane block could be an effective analgesic technique in patients undergoing laparoscopic liver resection. However, further prospective studies with large number of patients are needed to evaluate the effectiveness of this block in liver surgery.

Keywords: ESP block; Erector spinae plane block; Regional anesthesia ; Hepatic surgery; Pain management

\section{Introduction}

Laparoscopic liver surgery (LLS) has significant advantages compared with open surgery. These include reduced tissue trauma, decreased blood loss, and shorter length of hospital stay [1].

Despite these advantages, LLS can be associated with significant postoperative pain. Epidural analgesia (EA) is widely applied in liver surgery and blunts the perioperative neuroendocrine stress response, provides excellent postoperative pain relief and aids in early mobilization and postoperative rehabilitation.

However, due to concerns related to postoperative coagulopathy and potential risks and side effects of EA, the perceived benefit of it has been questioned in the context of the enhanced recovery after surgery (ERAS) recommendations in liver surgery [2].

Recently, the erector spinae plane (ESP) block has been considered a suitable interfascial plane block in thoracic and abdominal surgery thanks to its simple, secure and effective execution and, most of all, because it provides both visceral and somatic analgesia. It could become a good analgesic technique, as its effectiveness has been compared to thoracic EA [3-5]. This case series focuses on aspects of a continuous ESP block in LLS, which has not been previously reported in literature.

\section{Case Report}

Written informed consent was obtained from the patients and they were prospectively enrolled.

Patients were scheduled for left or right laparoscopic liver resection.

In the preoperative room, electrocardiogram, heart rate and pulse oximetry analysis were placed.

A FloTrac sensor was connected to an indwelling radial artery catheter and an EV1000/Volume View monitor (Edwards Lifesciences, Irvine, CA, USA), in order to obtain real-time blood pressure, cardiac index (CI), stroke volume index (SVI), and stroke volume variation (SVV).

The Bispectral Index (BIS) and train-of-four

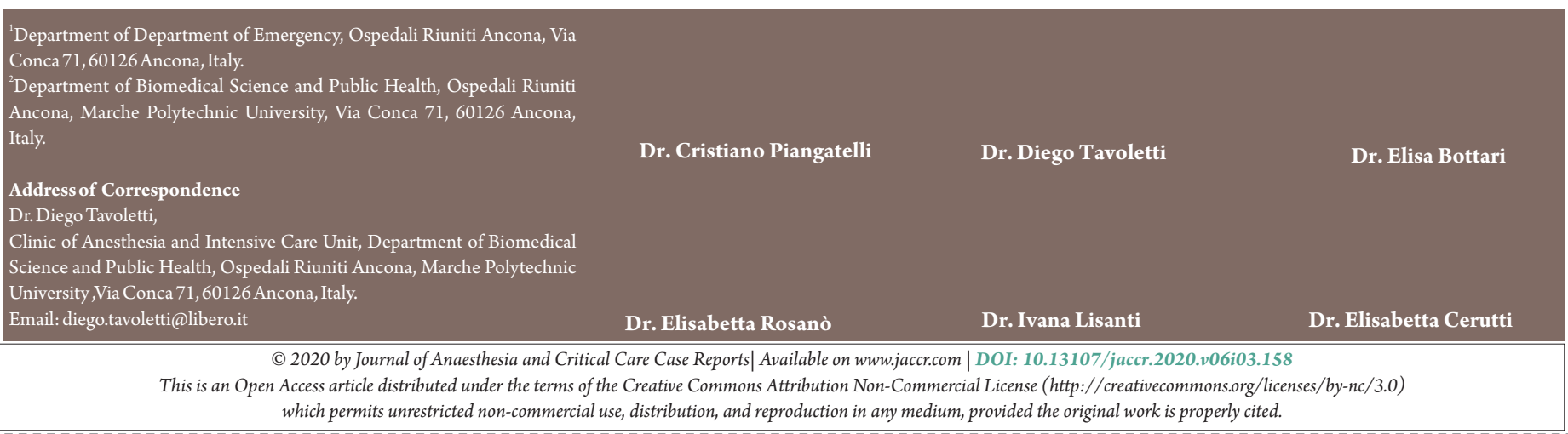

14 | Journal of Anaesthesia and Critical Care Case Reports | Volume 6; Issue 3 | September-December 2020 | Page 14-17 


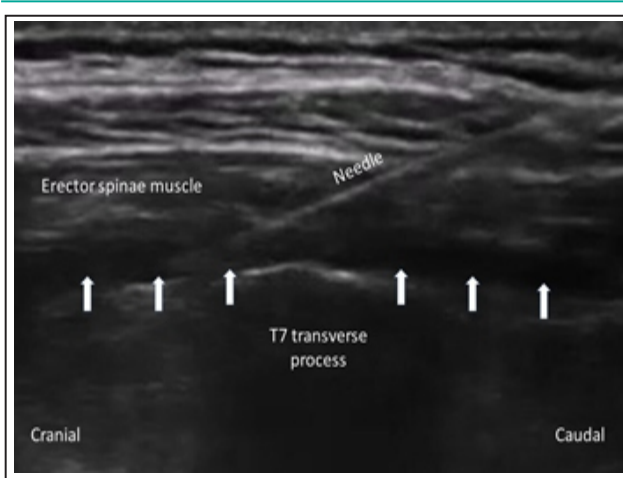

Figure 1: Ultrasound image of the erector spinae plane block at the level of T7. The arrows indicate the local anesthetic spread below the erector spinae muscle.

monitoring allowed to evaluate brain activity and neuromuscolar blockade, respectively.

All cases were performed under general anesthesia.

Anesthetic induction was accomplished with the intravenous administration of propofol, fentanyl, rocuronium and mantained with desflurane.

A triple lumen 14-gauge catheter was placed into one of the large internal jugular veins using ultrasound sonography in order to measure central venous pressure (CVP).

In all cases a combination of right continuous ESP block and transversus abdominis plane (TAP) plus oblique subcostal transversus abdominis plane (OSTAP) blocks in the left hemisome were performed for the surgical pain control. All blocks were performed using a low-frequency linear transducer (6-13 $\mathrm{MHz}$, Sonosite SII, Bothell, WA, USA). The patient was positioned in the left lateral decubitis position to allow the achievement of the ESP block. ESP block was carried out at the T7 level. The ultrasound probe was placed in the longitudinal orientation over the spinous process of the $7^{\text {th }}$ thoracic vertebra. The probe was then moved laterally to identify the tip of the right transverse process.

A $18 \mathrm{G}, 110 \mathrm{~mm}$ insulated needle (Contiplex; B. Braun Melsungen, Germany) was inserted in a ultrasound plane approach between erector spinae muscle and the transverse process.

To verify the correct needle positioning, hydrodissection was used with a total volume of $5 \mathrm{ml}$ of saline solution. Afterwards, a catheter was inserted and a bolus of $20 \mathrm{ml} 0.25 \%$ levobupivacaine was injected (Fig. 1). Then, the catheter was secured on the anterior thoracic wall.

Subsequently, TAP plus OSTAP blocks were performed contralaterally in according to Hebbard approach, injecting $20 \mathrm{ml}$ 0.25\% levobupivacaine in each procedure, for a total volume of $40 \mathrm{ml}[6]$.

EV $1000 /$ Volume View (Edwards Lifesciences, Irvine, CA, USA) parameters guided the intraoperative fluid and vasopressor/inotrope administration. The goal-directed fluid therapy was tailored to mantain $\mathrm{SVV}<15 \%, \mathrm{CI}>2,2 \mathrm{~L} / \mathrm{min} / \mathrm{m}^{2}$ and $\mathrm{CVP}<5 \mathrm{~cm} \mathrm{H}_{2} \mathrm{O}$. The CVP was measured to calculate systemic vascular resistance (SVR). The BIS value was maintained between 40 and 45 to assure a suitable level of anesthesia and the train-of-four monitoring secured the neuromuscularblock.

At the end of surgery, patients were extubated and transferred to the postoperative recovery room. Patients evaluated their surgical pain by using the Visual Analogue Scale (VAS) both at rest and after coughing and by giving a score from 0 to 10 in which 0 means no pain and 10 is the worst imaginable pain they have ever felt.

Surgical pain was evaluated until 48 hours after the operation.

Postoperative analgesia included an infusion of $0.25 \%$ levobupivacaine (injection velocity of $10 \mathrm{ml} / \mathrm{hr}$ ) into the ESP catheter for 36 hours and paracetamol 1 gm every 8 hours.

Results

Demographics and clinical data is summarised in table 1 .

Five patients received a right continuous ESP block and a left TAP plus OSTAP blocks for laparoscopic liver resection.

This chest and abdominal wall blocks combination was used to provide intra and postoperative analgesia.

During the intraoperative period, only fentanyl were administered as analgesic drug for tracheal intubation. Rapid increases in blood pressure or heart rate suggesting of poor analgesia have not been observed. Thus, no other analgesic procedures or drugs were administered during surgery.

Two patients reported intraoperative complications: patient no. 1 had sinus bradycardia during the carbon dioxide insufflation to achieve pneumoperitoneum, promptly resolved with administration of 0.5 $\mathrm{mg}$ of atropine, and patient no. 2 had two hypotensive states solved with administration of $3 \mathrm{mg}$ of ephedrine each time.

Postoperative pain scores over the first $48 \mathrm{hr}$ can be seen in table 2 . The highest pain scores at rest and after coughing are recorded at 48

\begin{tabular}{|c|c|c|c|c|}
\hline $\begin{array}{l}\text { Patient } \\
\text { no }\end{array}$ & Gender & Age (yrs) & Surgical Procedure & Co-morbidities \\
\hline 1 & Male & 54 & $\begin{array}{l}\text { Resection of the sixth liver } \\
\text { segment due to HCC }\end{array}$ & $\mathrm{HCV}$ \\
\hline 2 & Male & 79 & $\begin{array}{c}\text { Right hepatectomy due to } \\
\text { CRC metastasis }\end{array}$ & $\begin{array}{l}\text { CRF, DM, } \\
\text { CAD }\end{array}$ \\
\hline 3 & Male & 71 & $\begin{array}{c}\text { Resection of the fifth and } \\
\text { sixth liver segments due to } \\
\text { HCC }\end{array}$ & HBV, COPD \\
\hline 4 & Female & 58 & $\begin{array}{l}\text { Resection of the second } \\
\text { and third liver segments } \\
\text { due to CRC metastasis }\end{array}$ & None \\
\hline 5 & Female & 68 & $\begin{array}{c}\text { Left hepatectomy due to } \\
\text { HCC }\end{array}$ & PAF \\
\hline \multicolumn{5}{|c|}{$\begin{array}{l}\text { HCC: hepatocellular carcinoma, HCV: chronic hepatitis C virus } \\
\text { infection, CRC: colorectal cancer, CRF: chronic renal failure, DM: } \\
\text { diabetes mellitus, CAD: coronary artery disease, HBV: hepatitis B virus } \\
\text { infection, COPD: chronic obstructive pulmonary disease, PAF: } \\
\text { paroxysmal atrial fibrillation. }\end{array}$} \\
\hline
\end{tabular}

\begin{tabular}{|c|c|c|c|c|c|}
\hline \multicolumn{3}{||c|}{ VAble 2. Postoperative pain scores } \\
\hline $\begin{array}{c}\text { Time from the end } \\
\text { of surgery (hours) }\end{array}$ & $\begin{array}{l}\text { Patient } \\
\text { No.1 }\end{array}$ & $\begin{array}{l}\text { Patient } \\
\text { No.2 }\end{array}$ & $\begin{array}{l}\text { Patient } \\
\text { No.3 }\end{array}$ & $\begin{array}{l}\text { Patient } \\
\text { No.4 }\end{array}$ & $\begin{array}{l}\text { Patient } \\
\text { No.5 }\end{array}$ \\
\hline 0 & $0 / 0$ & $0 / 1$ & $0 / 1$ & $0 / 1$ & $0 / 1$ \\
\hline 1 & $0 / 1$ & $0 / 1$ & $0 / 2$ & $1 / 1$ & $0 / 1$ \\
\hline 3 & $0 / 0$ & $0 / 2$ & $0 / 1$ & $0 / 0$ & $0 / 2$ \\
\hline 6 & $1 / 1$ & $0 / 2$ & $0 / 0$ & $0 / 0$ & $0 / 1$ \\
\hline 12 & $0 / 0$ & $0 / 1$ & $0 / 0$ & $0 / 0$ & $0 / 1$ \\
\hline 24 & $1 / 1$ & $1 / 1$ & $1 / 1$ & $0 / 0$ & $0 / 0$ \\
\hline 36 & $0 / 1$ & $0 / 1$ & $1 / 1$ & $0 / 0$ & $0 / 1$ \\
\hline 48 & $2 / 2$ & $0 / 2$ & $1 / 2$ & $1 / 1$ & $1 / 2$ \\
\hline VAS: visual analogue scale & & & & & \\
\hline
\end{tabular}


hr. No patient required additional doses of analgesia.

Postoperative recoveries of all patients were uneventful and all of them were discharged within 5 days after surgery.

\section{Discussion}

LLS is a modern treatment of choice for most liver cancers and is a less invasive technique than open surgery. This reduced postoperative complications including pain, accelerated patient recovery and shortened hospitalization [7].

However, LLS can cause painful conditions that require adequate pain management.

Ultrasound guided ESP block has been used to provide postoperative analgesia in many painful conditions such as breast, thoracic, bariatric and abdominal surgery $[3-5,8,9]$.

The mechanism of action of ESP block and its pain releasing effect is still under study.

Some authors claim that the somatic and visceral analgesia caused by this blockage is due to a diffusion of the local anesthetic in the paravertebral space, demonstrated through resonance imaging. Similarly, other authors affirmed this in cadavers and in living patients.

In fact Adhikary et al. used magnetic resonance imaging to evaluate the diffusion of contrast on three cadavers. In all of them paravertebral diffusion was observed [10]. While in two other studies, the paravertebral spread was detected on living patients [11, 12]. Nevertheless, in one study, ESP block was performed in two cadavers. Subsequently, multi-slice computed tomography was performed to evaluate radiocontrast distribution. The authors conclude that the contrast did not spread to the paravertebral space. However, they also claimed that the computed tomography offers less spatial resolution and soft tissue definition than magnetic resonance imaging used as a method of investigation in other studies [13].

Concerning the local anesthetic diffusion on the longitudinal axis, a craniocaudal distribution has been observed along the trunk. It is influenced by the injection level and the local anesthetic volume. In previous studies, the injection of $20 \mathrm{ml}$ of fluid at the T7 level determines a diffusion from the upper thoracic vertebral segments to the lumbar ones [13]. In favor of this, clinical investigations reported that the ESP block at $\mathrm{T} 7$ level resulted in a postoperative pain relief after major surgery $[4,14,15]$.

Pain following abdominal surgery has two components: somatic pain and visceral pain.

In our case series, for the intra and postoperative somatic/visceral pain relief, we used a combination of continuous ESP block on one side and TAP plus OSTAP blocks on the other side.

In the right side, the somatic pain (due to skin incision and laparoscopic ports insertion) and visceral pain (caused by the liver parenchimal resection) were covered by the right ESP block, while on the left side we had to cover only somatic pain (due to skin incision and laparoscopic ports insertion) and not visceral pain, because there wasn't visceral resection.

In this way, we considered that this combination could provide adequate intra and postoperative analgesia.

A bilateral ESP block could have been an alternative to our choice. However, as explained previously, the mechanism of action of this block is not yet clear, and a possible bilateral diffusion of the local anesthestic within the paravertebral space, could cause hypotension due to the autonomic nervous system block.

In the ERAS pathways for abdominal surgery, EA is a key element as it allows to reduce the stress response to surgery in the perioperative period and has an opioid-sparing effect within the first $48 \mathrm{~h}$ postoperatively. Despite these benefits, its role and safety have been questioned because of the following problems: postoperative coagulopathy and hypotension. After liver resection, an increase in prothrombin time and a decrease in platelet counts can be detected from the first to the fifth postoperative day. This can increase the risk of epidural hematoma after catheter removal. Blockage of the autonomic nervous system leads to hypotension, which results in a decrease in splanchnic perfusion. In cirrhotic patients, with pre-existing diseases, it leads to organ dysfunction in the postoperative period. In particular, hypotension leads to a reduction in renal perfusion with an increased risk of acute kidney injury. Moreover, evidence has shown that patients with EA need an increased administration of fluids and vasopressor support. Another reason why the EA is under investigation concerns its true need in the field of laparoscopy. This surgical technique is associated with less postoperative pain than open surgery. The analgesia provided by the epidural block may be more than necessary [7].

In liver disease, drug metabolism may be reduced, especially in cirrhosis. This leads to an increase in the bioavailability and accumulation of the drug. In addition, hepatic resection reduces liver volume, contributing to the decrease of pharmacological metabolism. Therefore, opioids and nonsteroidal anti-inflammatory should be used cautiously [7].

\section{Conclusion}

Thoracic and abdominal wall blocks could be an effective analgesic technique for postoperative pain management. ESP block is a simple and very useful technique for analgesia in abdominal surgery. Based on our findings, it provided pain relief for 48 hours, without the need additional dose of analgesia. This suggests that ESP block may be recommended as an alternative for pain management in liver surgery.

Further investigation it would be necessary to determine the advantages and disadvantages of this technique compared to other regional or systemic analgesic approaches.

\section{References}

1. Savikko J, Vikatmaa L, Hiltunen A, Mallat N, Tukiainen E, Salonen SM, et al. Enhanced recovery protocol in laparoscopic liver surgery. Surg Endosc. 2020.

2. Melloul E, Hübner M, Scott M, Snowden C, Prentis J, Dejong CHC, et al. Guidelines for perioperative care for liver surgery: Enhanced Recovery After Surgery (ERAS) Society recommendations. WorldJ Surg. 2016;40:2425-40.

3. Forero M, Adhikary SD, Lopez H, Tsui C, Chin KJ. The Erector Spinae Plane Block: a novel analgesic technique in thoracicneuropathic pain. Reg Anesth Pain Med.2017;41(5):621-7.

4. Chin KJ, Malhas L, Perlas A. The Erector Spinae Plane Block provides visceral abdominal analgesia in bariatric surgery: a report of 3 cases. Reg Anesth Pain Med. 2017;42(3):372-6.

5. Restrepo-Garces CE, Chin KJ, Suarez P, DiazA. Bilateral Continuous Erector Spinae Plane Block Contributes to Effective Postoperative Analgesia After Major Open Abdominal Surgery. A \& A Case Reports. 2017;9:319-21.

6. Hebbard, P. Subcostal transversus abdominis plane block under ultrasound guidance. Anesth Analg. 2008;106(2):674-75.

7. Agarwal, V, Divatia, J. (2019) Enhanced Recovery after Surgery in Liver Resection: Current Concepts and Controversies. Korean J Anesthesiol.2019; 72:119-29. 
8. Ueshima H, Otake H. Erector spinae plane block provides effective pain management during pneumothorax surgery.JClin Anesth. 2017;40:74.

9. Bonvicini D, Tagliapietra L, Giacomazzi A, Pizzirani E. Bilateral ultrasound-guided erector spinae plane blocks in breast cancer and reconstruction surgery.J Clin Anesth. 2017;44:3-4.

10. Adhikary SD, Bernard S, Lopez H, Chin KJ. Erector spinae plane block versus retrolaminar block: a magnetic resonance imaging and anatomical study. Reg Anesth Pain Med. 2018;43:756-62.

11. Schwartzmann A, Peng P, Maciel MA, Forero M. Mechanism of the erector spinae plane block: insightsfrom a magnetic resonance imaging study. CanJ Anesth. 2018; 65:1165-6.
12. Ueshima H, Hiroshi O. Spread of local anesthetic solution in the erector spinae plane block. JClin Anesth. 2018;45:23.

13. Aponte A, Sala-Blanch X, Prats-Galino A, Masdeu J, Moreno LA, Sermeus LA. Anatomical evaluation of the extent of spread in the erector spinae plane block: a cadaveric study. Can J Anesth. 2019;66:886-93.

14. Tulgar S, Selvi O, Kapakli MS. Erector spinae plane block for different laparoscopic abdominal surgeries: case series. Case Rep Anesthesiol 2018;2018.

15. Niraj G, Zubair T. Continuous Erector Spinae Plane (ESP) Analgesia In Different Open Abdominal Surgical Procedures: A Case Series. JAnesth Surg. 2018;5:57-60.
Conflict of Interest: Nil

Source of Support: None
How to Cite this Article

Piangatelli C, Tavoletti D, Bottari E, Rosanò E, Lisanti I, Cerutti E | Erector Spinae Plane Block- An Analgesic Technique for Laparoscopic Liver Resection: A Case Series | Journal of Anaesthesia and Critical Care Case Reports | September-December 2020; 6(3): 14-17. 